\title{
Verzeichnis der Mitglieder der Vereinigung der Deutschen Staatsrechtslehrer
}

Stand: 1.1 .1986

\section{Vorstand}

1. Rupp, Dr. Hans Heinrich, o. Professor, Am Marienpfad 29, 6500 Mainz, (06131) 34588

2. Häberle, Dr. Peter, o. Professor Universität Bayreuth, Universitätsstraße, Postfach 3008, 8580 Bayreuth, (09 21) 552947

3. Erichsen, Dr. Hans-Uwe, o. Professor, Falkenhorst 17, 4400 Münster-St. Mauritz, (02 51) 31312 Univ. Münster, (02 51) 832741

\section{Mitglieder}

1. Abelein, Dr. Manfred, o. Professor, Rheinweg 12, 5300 Bonn, (02 28) 25692 (Universität Regensburg)

2. Achterberg, Dr. Norbert, o. Professor, Küperweg 11, 4400 Münster, (0 25 34) 74 22; Univ., (02 51) 832761

3. Adamovich, Dr. Ludwig, o. Professor a. D., Roosevelt-Platz 4, A-1090 Wien, (02 22) 4273 95; dienstl., (02 22) 66162361

4. Alexy, Dr. Robert, Privatdozent, Düstere Eichen Weg 56, 3400 Göttingen, (05 51) 41187 Univ., (05 51) 397383

5. Antoniolli, Dr. Dr. h. c. Walter, o. Universitätsprofessor, Ottensteinstr. 35, A-2344 Maria Enzersdorf, (0 22 36) 45 09; (Universität Wien)

6. Armbruster, Dr. Hubert, o. Professor, An der Allee 69, 6500 Mainz, (061 31) 319 50; Univ., (0 61 31) 392384 
7. Arndt, Dr. Hans-Wolfgang, o. Professor, Waldstr. 34, 6730 Neustadt/Weinstraße (0 63 21) 33385 Universität Mannheim, (06 21) 2925195

8. v. Arnim, Dr. Hans Herbert, o. Professor, Im Oberkämmerer 26, 6720 Speyer, (0 62 32) 981 23; Hochschule Speyer, (0 62 32) 910343

9. Arnold, Dr. Rainer, o. Professor, Wolfsteinerstr. 14, 8400 Regensburg, (09 41) 996 70; Univ., (09 41) $9432654 / 5$

10. Baade, Dr. Hans W., Professor, 6002 Mountain Climb Drive, Austin/Texas, USA, 78731 , Tel. (512) 4525071 und 4715151

11. Bachof, Dr. Dr. h. c. Otto, o. Professor, Auf dem Kreuz 3, 7400 Tübingen, (0 70 71) 611 44; Univ., (0 70 71) 294910 od. 292549

12. Badura, Dr. Peter, o. Professor, Am Rothenberg Süd 4, 8113 Kochel am See, (0 88 51) 5289 Universität München

13. Barbey, Dr. Günther, apl. Professor, Stallupöner Allee 22, 1000 Berlin 19, (0 30) 3055703

14. Barfuß, Dr. iur. Dr. rer. pol. Walter, a. o. Universitätsprofessor, Tuchlauben 13, A-1014 Wien, (02 22) 638761

15. Bartlsperger, Dr. Richard, o. Professor, Schleifweg 55, 8521 Uttenreuth, (0 91 31) 599 16; Universität Erlangen, (0 91 31) 852818

16. Battis, Dr. Ulrich, Professor, Rummenohler Str. 91, 5800 Hagen (0 23 55) 21 55;

Fernuniv., (0 23 31) 8042917

17. Bayer, Dr. Hermann-Wilfried, Professor, Henkenbergstr. 45a, 4630 Bochum, (02 34) 7917 44; Univ., (02 34) 7005724

18. Becker, Dr. Jürgen, Privatdozent, Unterer Mühlenweg 75, 7800 Freiburg, (07 61) 4455 79; Univ., (07 61) 2033567

19. Berchtold, Dr. Klaus, Universitätsdozent, Bräunerstr. 4-6/22, A-1010 Wien, (02 22) 531434

20. Berg, Dr. Wilfried, o. Professor, Waldsteinring 25, 8580 Bayreuth, (09 21) 931 25; Univ., (09 21) 552876 
21. Berka, Dr. Walter, Universitätsdozent, Birkenweg 2, A-5400 Hallein, (0 62 45) 625 52; dienstl.: Weiserstr. 22, A-5020 Salzburg, (06 62/4 4511$) 316$

22. Bernhardt, Dr. Rudolf, o. Professor, Gustav-Kirchhoff-Str. 2a, 6900 Heidelberg, (0 62 21) 436 99; dienstl., (0 62 21) 4 82-1

23. Bethge, Dr. Herbert, o. Professor, Am Seidenhof 10, 8390 Passau, (08 51) 416 97; Univ., (08 51) 509197

24. Bettermann, Dr. Dr. h. c. Karl-August, o. Professor, Alte Landstr. 173, 2000 Hamburg 63, (0 40) 53840 64; Univ., (0 40) 41234557

25. Binder, Dr. Bruno, Universitätsdozent, Mozartstr. 1, A-4020 Linz, (07 32) 275110 oder Wischerstr. 30, A-4020 Linz, (07 32) 239926. Univ. Linz, Altenbergerstr. 69, A-4040 Linz (07 32) 231381411

26. Birk, Dr. Dieter, o. Professor, Kinderhauser Str. 6, 4400 Münster, (02 51) 2729 82; Univ., (02 51) 832795

27. Blankenagel, Dr. Alexander, Privatdozent, Univ. Bayreuth, Universitätsstraße, Postfach 3008 , 8580 Bayreuth, (09 21) 552946

28. Bleckmann, Dr. Dr. Albert, o. Professor, Straßburger Weg 44, 4400 Münster, (02 51) 7960 00; Univ., (02 51) 832021

29. Blümel, Dr. Willi, o. Professor, Angelhofweg 65, 6901 Wilhelmsfeld, (0 62 20) 18 80; Hochschule Speyer, (0 62 32) 106362

30. Blumenwitz, Dr. Dieter, o. Professor, Herzog-Albrecht-Str. 26, 8011 Zorneding, (0 81 06) 26 82; Universität Würzburg, (09 31) 31308

31. Böckenförde, Dr. iur. Dr. phil. Ernst-Wolfgang, o. Professor, Türkheimstr. 1, 7801 Au bei Freiburg, (07 61) 4056 23; Universität Freiburg, (07 61) 2033431

32. Böckstiegel, Dr. Karl-Heinz, Professor, Parkstr. 38, 5060 Bergisch-Gladbach, (0 22 04) 662 68; Universität Köln, (02 21) 4702337 
33. Bogs, Dr. Harald, Professor, Dresdener Str. 7, 3406 Bovenden, (05 51) 815 95; Universität Göttingen, (05 51) 397392

34. Bothe, Dr. Michael, Professor, Theodor-Heuss-Str. 6, 6140 Bensheim, (06 251) 4345 Univ. Frankfurt a.M. 1, (069) 7982264

35. Breuer, Dr. Rüdiger, Professor, Heinrich-Brauns-Str. 4, 5500 Trier, (06 51) 214 78; Univ., (06 51) 2012578

36. Brohm, Dr. Winfried, o. Professor; Wydenmööslistr. 11, CH-8280 Kreuzlingen, (0 72) 751525 ; Universität Konstanz, (0 75 31) 8821 69/76

37. v. Brünneck, Dr. Alexander, Privatdozent, Blumenhagenstr. 5, 3000 Hannover 1,(05 11) 716911 Universität Hannover, (05 11) 4738228

38. Brünner, Dr. Christian, o. Universitätsprofessor, Rosengasse 9, A-8042 Graz, (03 16) 445 18; Univ., (03 16) $31581 / 476$

39. Brunner, Dr. Georg, o. Professor, Schloßstr. 7, 5014 Kerpen-Horrem, (0 22 73) 47 56; Univ. Köln, Ubierring 53, 5000 Köln 1, (02 21) 315110 u. 315149

40. Bryde, Dr. Brun-Otto, Professor, Connollystr. 15, 8000 München 40, (0 89) 35124 65; Hochschule der Bundeswehr München, Werner-HeisenbergWeg 39, 8014 Neubiberg (0 89) 60 04/42 40 oder 4241

41. Bull, Dr. Hans-Peter, o. Professor, Waldingstr. 35, 2000 Hamburg 65, (0 40) 64004 33; dienstl. Univ. Hamburg, (0 40) 41235760

42. Bullinger, Dr. Martin, o. Professor, Altschlößleweg 4, 7801 Au bei Freiburg, (07 61) 4023 89; Universität Freiburg, (07 61) 2033516

43. Burmeister, Dr. Joachim, o. Professor, Blücherstr. 37, 6670 St. Ingbert-Rohrbach, (0 68 94) 575 83; Univ., (06 81) 3022128

44. v. Campenhausen, Dr. Frhr. Axel, Professor, Goßlenstr. 109, 3400 Göttingen; Univ., (05 51) 397368

45. Carstens, Dr. Karl, o. Professor, Dechant-Kreiten-Str. 43, 5309 Meckenheim, (0 22 25) 2455 
46. Dagtoglou, Dr. Prodromos, o. Professor,

Hippokrates Str. 33, Athen 144/Griechenland,

Universität Athen, 00 30/1/362 9065

47. Degenhart, Dr. Christoph, Professor,

Stormstr. 3, 8500 Nürnberg 20,(09 11) 5924 62;

Univ. Münster, (02 51) 839803

48. Delbrück, Dr. Jost, Professor,

Schoolredder 20, 2300 Kiel-Altenholz, (04 31) 3225 58;

Univ., (04 31) 8802149

49. Denninger, Dr. Erhard, Professor,

Am Wiesenhof 1,6240 Königstein 3,(061 73) 789 32;

Universität Frankfurt, (0 69) 7982654

50. Dicke, Dr. Detlev Christian, o. Professor,

Alpenstr. 919, CH-3178 Bösingen

Universität Freiburg/Schweiz

51. Dittmann, Dr. Armin, o. Professor,

Süsserstr. 34, 7400 Tübingen 9, (0 70 71) 824 56;

Universität Hohenheim-Schloß, Postf. 7005 62,

7000 Stuttgart 70, (07 11) 45 01-2791

52. Doehring, Dr. Karl, o. Professor,

Mühltalstr. 117/3, 6900 Heidelberg, (0 62 21) 458 80;

Univ., (0 62 21) 547454

53. Dolzer, Dr. Dr. Rudolf, Privatdozent,

Am Pferchelhang 4/1, 6900 Heidelberg, (0 62 21) 8033 44;

Max-Planck-Institut für Völkerrecht, Berliner Str. 48, 6900 Heidelberg 1, (0 62 21) 482252

54. Dreier, Dr. Ralf, o. Professor,

Wilhelm-Weber-Str. 4, 3400 Göttingen, (05 51) 591 14;

Univ., (05 51) 397384

55. Dürig, Dr. Günter, o. Professor,

Staufenstr. 9, 7400 Tübingen, (0 70 71) 82508

56. Eberle, Dr. Carl-Eugen, Professor,

Ohnhorststr. 22, 2000 Hamburg 52, (0 40) 828640

Univ., (0 40) 41 23-3503

57. Ebsen, Dr. Ingwer, Professor, Mühltalstr. 24, 6500 Mainz 21, (0 61 31) 471169 ;

Univ. Münster, (02 51) 832718

58. Ehlers, Dr. Dirk, Professor,

Am Mühlenbach 14, 4403 Senden, (0 25 97) 84 15;

Universität Münster, (02 51) 832745 
59. Ehmke, Dr. Horst, o. Professor, Bundeshaus, 5300 Bonn 1, (02 28) 163429 oder 164834

60. Eichenberger, Dr. Dr. h. c. Kurt, o. Professor, Bärenbrunnenweg 4, CH-4144 Arlesheim b. Basel, (0 61) 723386

61. Erbel, Dr. Günter, Professor, Moselweg 3, 5300 Bonn 1; Univ., (02 28) 735583

62. Erichsen, Dr. Hans-Uwe, o. Professor, Falkenhorst 17, 4400 Münster-St. Mauritz, (02 51) 313 12; Universität Münster, (02 51) 832741

63. Ermacora, Dr. Felix, o. Universitätsprofessor, Karl-Lueger-Ring, A-1010 Wien I, (02 22) 4276 11; Univ., (02 22) 430031 45, Schottenbastei 10-16

64. Evers, Dr. Hans-Ulrich, o. Universitätsprofessor, Wolfsgartenweg 30, A.5020 Salzburg, (06 62) 2602 03; Univ., (06 62) $44511 / 316$

65. Faber, Dr. Heiko, Professor, Wunstorfer Str. 1, 3007 Gehrden 1, (0 51 08) 22 34; Universität Hannover, (05 11) 4738206

66. Fiedler, Dr. Wilfried, o. Professor, Holm 27, 2300 Kiel-Rammsee, (04 31) 655 69; Univ. des Saarlandes, Fachbereich I, Im Stadtwald, 6600 Saarbrücken, (06 81) 3023200

67. Fleiner-Gerster, Dr. Dr. h.c. Thomas, o. Professor, Le Riedelet 9, CH-1723 Marly FR, (0 37) 461261

68. Folz, Dr. Hans-Ernst, Professor, An der Haustatt 50, 3550 Marburg, (0 64 21) 635 36; Univ., (0 64 21) 2831 23/28

69. Frank, Dr. Götz, Professor, Friedrichstr. 52, 3257 Springe 1; Universität Hannover, (05 11) 47382 08/82 29

70. Friauf, Dr. Karl Heinrich, o. Professor, Eichenhainallee 17, 5060 Bergisch-Gladbach 1, (0 22 04) 61984

71. Fröhler, Dr. Ludwig, o. Universitätsprofessor, Altebergerstr. 39, A-4010 Linz-Urfahr 
72. Fromont, Dr. Dr. h. c. Michel, Professor, 9, Impasse Henri Bouchard, F-21000 Dijon, (80) 5741 65; Univ., (80) 668134

73. Frotscher, Dr. Wemer, o. Professor, Habichtstalgasse 32, 3550 Marburg/Lahn, (0 64 21) 32961 Univ., Universitätsstr. 6, (0 64 21) 283122

74. Frowein, Dr. Jochen A., o. Professor, Berliner Str. 48, 6900 Heidelberg 1 dienstl., (0 62 21) 4 82-1

75. Funk, Dr. Bernd-Christian, o. Professor, Mariatroster Str. 111/3, A-8045 Graz, (03 16) 310 82; Univ., (03 16) 31581

76. Gallent, DDr. Kurt, Professor, Senatsrat, Pestalozzistr. 1/III, A-8010 Graz, (03 16) 7789 62; dienstl., (03 16) 73054

77. Gallwas, Dr. Hans-Ullrich, Professor, Hans-Leipelt-Str. 16, 8000 München 40, (0 89) 3283 66; Univ., (0 89) 21803262

78. Geck, Dr. Dr. h. c. Wilhelm Karl, M. A., o. Professor, Privatweg, 6670 St. Ingbert-Reichenbrunn, (0 68 94) 73 26; Universität Saarbrücken, (06 81) 3022105

79. Göldner, Dr. Detlef, Privatdozent, Wilhelmshavener Str. 20, 2300 Kiel, (04 31) 81644

80. Görg, Dr. Hubert, o. Professor, Ellersberg, 5064 Rösrath, (0 22 05) 2540

81. Goerlich, Dr. Helmut, Privatdozent, Dorotheenstr. 95, 2000 Hamburg 60, (0 40) 2798706 dienstl., (0 40) 34 97-3890

82. Götz, Dr. Volkmar, o. Professor, Geismarlandstr. 17a, 3400 Göttingen, (05 51) 43119

83. Grabitz, Dr. Eberhard, o. Professor, Cosimaplatz 2, 1000 Berlin 41, (0 30) 8522136 ; Univ., (0 30) 8384949

84. Gramlich, Dr. Ludwig, Privatdozent, Winterleitenweg 1c, 8700 Würzburg, (09 31) 8812 32; Univ., (09 31) 31334

85. Grawert, Dr. Rolf, o. Professor, Aloysiusstr. 28, 4630 Bochum 1, (02 34) 4736 92; Univ., (02 34) 7002809 
86. Grewe, Dr. Dr. h. c. Wilhelm G., o. Professor, Zum Kleinen Ölberg 28, 5330 Königswinter 41 (Thomasberg), (0 22 44) 68 74; dienstl., (02 28) 214160

87. Grimm, Dr. Dieter, o. Professor, Fahlenbreede 2, 4802 Halle, (0 52 01) 102 40; Universität Bielefeld, (05 21) 1064405

88. Grupp, Dr. Klaus, Privatdozent, Ostpreußenring 6, 6830 Schwetzingen, (0 62 02) 17 193;

Hochschule für Verwaltungswissenschaften, Freiherr-vom-Stein-Str. 2, 6720 Speyer, (06 232) 910395

89. Gygi, Dr. Fritz, o. Professor, Beatusstr. 28, CH-3006 Bem, (0 31) 448638

90. Häberle, Dr. Peter, o. Professor, Universität Bay reuth, Universitätsstraße, Postfach 3008 , 8580 Bayreuth, (09 21) 552947

91. Häfelin, Dr. Ulrich, o. Professor, Müseliweg 1, CH-8049 Zürich, (01) 568460

92. Hahn, Dr. Hugo J., LL. M. (Harvard), o. Professor, Frankenstr. 63, 8700 Würzburg 1, (09 31) 2842 86; Univ., (09 31) 31310

93. Hailbronner, Dr. Kay, o. Professor, Toggenbühl, CH-8557 Fruthwilen, (0 72) 64 19 46; Universität Konstanz, (0 75 31) 882247

94. Haller, Dr. Herbert, a. o. Universitätsprofessor, Felix-Mottl-Str. 48 Haus 2, A-1 190 Wien, (02 22) 341 72 14; Univ., (02 22) 347541

95. Haller, Dr. Walter, o. Professor, Burgstr. 264, CH-8706 Meilen, (01) 92310 14; Universität Zürich, (01) 2573003

96. Hangartner, Dr. Yvo, o. Professor, Am Gozenberg 2, CH-9202 Gossau, (0 71) 851511

97. Haverkate, Dr. Görg, Professor, Fürst-Pückler-Str. 70, 5000 Köln 41, (02 21) 4010 84; Universität Frankfurt/M., (0 69) 7 98-27 03

98. Heckel, Dr. Martin, o. Universitätsprofessor, Lieschingstr. 3, 7400 Tübingen, (0 70 71) 614 27; Univ., (0 70 71) 292971 
99. Hendler, Dr. Reinhard, Professor, Im Baumgarten 8, 7750 Konstanz 16, (0 75 31) 44609 Universität Konstanz, (0 75 31) 88-27 55 od. 2328

100. Hengstschläger, Dr. Johann, Universitätsprofessor, Auf der Halde 16, A-4020 Linz; Univ., (0 72 22) 31381

101. Henke, Dr. Wilhelm, o. Professor, Laufer Str. 5, 8501 Rückersdorf, (0 91 23) 2785

102. Hermann, Dr. Günter, Professor, Weiherstr. 34, 5303 Bornheim 3, (0 22 27) 5772

103. Herzog, Dr. Roman, o. Professor, Ludwigstr. 35, 7024 Filderstadt 1; dienstl., (07 11) 20723100

104. Hesse, Dr. Dr. h. c. Konrad, o. Professor, Schloßweg 29, 7802 Merzhausen, (07 61) 4038 11; Universität Freiburg, (07 61) 2033514

105. Hettlage, Dr. Karl-Maria, o. Professor, Friedrich-Ebert-Str. 83, 5300 Bonn-Bad Godesberg, (02 28) 364361

106. Frhr. v. d. Heydte, Dr. jur., Dr. rer. pol. Friedrich-August, o. Professor, Hagschneiderweg 1, 8311 Aham-Vils, (0 87 44) 1064

107. Heyen, Dr. Erk Volkmar, Privatdozent, Landauer Warte 1, 6720 Speyer, (0 62 32) 957 48; Hochschule, (0 62 32) 910346

108. Hilf, Dr. Meinhard, o. Professor, Schelpsheide 12, 4800 Bielefeld 1, (05 21) 8892 82; Univ., (05 21) 1 06-44 03

109. Hill, Dr. Hermann, Privatdozent, Rotdornweg 21, 2303 Gettorf, (0 43 46) 67 14; Lorenz-vom-Stein-Inst. für Verwaltungswissenschaften, Univ. Kiel, Olshausenstr. 40-60, Haus N 61c, 2300 Kiel, (04 31) 8804540

110. Höhn, Dr. Emst, o. Professor, Wiesenstr. 6, CH-9302 Kronbühl, (0 71) 255146 (Hochschule St. Gallen)

111. Hoffmann, Dr. Gerhard, o. Professor, Ernst-Lemmer-Str. 10/I, 3550 Marburg 6-Wehrda, (064 21) 81645 
112. Hoffmann-Riem, Dr. Wolfgang, Professor,

Kätnerweg 24, 2000 Hamburg 65, (0 40) 64024 78;

Univ., (0 40) 41235416

113. Hofmann, Dr. Hasso, o. Professor,

Christoph-Mayer-Weg 5, 8700 Würzburg, (09 31) 873 88;

Univ., (09 31) 31336

114. Hollerbach, Dr. Alexander, o. Professor,

Parkstr. 8, 7806 March-Hugstetten, (0 76 65) 22 51;

Universität Freiburg, (07 61) 2033535

115. Hoppe, Dr. Werner, o. Professor,

Linckensstr. 131, 4400 Münster, (0 25 01) 82 27;

dienstl., (02 51) 832703

116. Huber, Dr. Ernst Rudolf, o. Professor, In der Röte 2, 7800 Freiburg, (07 61) 53713

117. Huber, Dr. Dr. h. c. Hans, o. Professor, Mannenriedstr. 5, CH-3074 Muri b. Bem, (0 31) 520925

118. Hufen, Dr. Friedhelm, Professor, Römerauterrasse 5, 8910 Landsberg/Lech, (0 81 91) 395 38;

Universität Augsburg, (08 21) 598429

119. Ipsen, Dr. Hans Peter, o. Professor, Augustinum App. 1142, 2410 Mölln, (0 45 42) 813142 oder

Seminaris App. 1, 2120 Lüneburg, (0 4131$) 401131$

120. Ipsen, Dr. Jörn, o. Professor, Luisenstr. 41, 4550 Bramsche, (0 54 61) 44 96;

Universität Osnabrück, (05 41) 6 08-61 58/61 69

121. Ipsen, Dr. Knut, o. Professor, Nevelstr. 59, 4630 Bochum-Weitmar, (02 34) 4312 66;

Univ., (02 34) 7002820

122. Isensee, Dr. Josef, o. Professor, Weberstr. 98, 5300 Bonn 1,(02 28) 219166 ;

Univ., (02 28) 737983

123. Jaag, Dr. Tobias, Privatdozent, Abeggweg 15, CH-8057 Zürich, (01) 2112550

124. Jaenicke, Dr. Günther, Professor, Waldstr. 13,6906 Leimen b. Heidelberg, (0 62 24) 3571 (Universität Frankfurt)

125. Jahreiß, Dr. jur., Dr. h. c. mult., Hermann, o. Professor, Nasse-Str. 30, 5000 Köln 41, (02 21) 4615 53;

Univ., (02 21) 4702266 
126. Jakob, Dr. Wolfgang, o. Professor, Wilhelmstr. 25, 8000 München 40, (0 89) 390506

(Universität Augsburg)

127. Jarass, Dr. Hans D., LL.M. (Harvard), o. Professor, Kulmer Str. 12, 4630 Bochum 1, (02 34) 311291 ; Univ. Bochum, Postfach 1021 48,(02 34) 7002818

128. Kaiser, Dr. jur., Dr. rer. pol. h. c. Joseph H., o. Professor, Rothofweg, 7813 Staufen i. Br., (0 76 33) 57 28;

Universität Freiburg, (07 61) 2033567

129. Karpen, Dr. Ulrich, Professor, Hahnenstr. 19, 5030 Huerth, (0 22 33) 646 90; Universität Hamburg

130. Kewenig, Dr. Wilhelm, o. Professor, Schützallee 37, 1000 Berlin 37, (0 30) 80284 87;

Univ., (0 30) 3032316

131. Khol, Dr. Andreas, Universitätsprofessor, Cuviergasse 23, A-1130 Wien, (02 22) 8415 73; dienstl., (02 22) 831531

132. Kimminich, Dr. Otto, o. Professor, Killermannstr. 6, 8400 Regensburg, (09 41) 328 54; Univ., (09 41) 9432660

133. Kipp, Dr. Heinrich, Universitätsprofessor, Lanserstr. 61, A-6080 Igls, (0 52 22) 7209

134. Kirchhof, Dr. Ferdinand, Privatdozent, Wiesenweg 1, 6903 Neckargemünd 3, (0 62 23) 25 59;

Hochschule für Verwaltungswissenschaften, Freiherr-vom-Stein-Str. 2, 6720 Speyer, (062 32) 910387 oder 910386

135. Kirchhof, Dr. Paul, o. Professor, Am Pferchelhang 33/1, 6900 Heidelberg 1, (0 62 21) 8014 47; Univ., (062 21) 547457

136. Kirn, Dr. Michael, o. Professor, Rummelburgerstr. 5, 2000 Hamburg 73, (0 40) 64738 42; Hochschule der Bundeswehr, (0 40) 65412782

137. Kisker, Dr. Gunter, o. Professor, Waldstr. 74, 6301 Linden-Am Mühlberg, (0 64 03) 6 10 30; Universität Gießen, (06 41) 7025025 
138. Klecatsky, Dr. Hans R., o. Universitätsprofessor, Reithmannstr. 20, A-6020 Innsbruck, (0 52 22) 4676 74; Univ., (0 52 22) 336 01/7 33

139. Klein, Dr. Eckart, o. Professor, Ebersheimer Weg 35, 6500 Mainz, (0 61 31) 53670

140. Klein, Dr. Hans Hugo, o. Professor, Heilbrunnstr. 4, 7507 Pfinztal-Söllingen, (0 72 40) 7300 Universität Göttingen, (05 51) 394625

141. Kloepfer, Dr. Michael, o. Professor, Südallee 37A, 5500 Trier, (06 51) 419 32; Univ., (06 51) 201-25 56

142. Knemeyer, Dr. Franz-Ludwig, o. Professor, Unterdürrbacher Str. 353, 8700 Würzburg, (09 31) 961 18; Univ., (09 31) 31899

143. Knies, Dr. Wolfgang, o. Professor, Am Botanischen Garten 5, 6600 Saarbrücken 11; Univ., (06 81) 3022104

144. Knöpfle, Dr. Franz, o. Professor, Höhenweg 22, 8901 Leitershofen; Universität Augsburg, (08 21) 598352

145. Koch, Dr. Hans-Joachim, Professor, Wendlohstr. 80, 2000 Hamburg 61, (0 40) 55188 04; Univ., (0 40) 41233977

146. König, Dr. Dr. Klaus, Professor, Wimphelingstr. 5, 6720 Speyer, (0 62 32) 59 01; dienstl. Adenauerallee 141, 5300 Bonn, (02 28) 562300

147. Kopp, Dr. Ferdinand O., o. Universitätsprofessor, Innstr. 40, 8390 Passau

148. Korinek, Dr. Karl, o. Universitätsprofessor, Auhofstr. 225, A-1130 Wien, (02 22) 82091 53; Univ., (02 22) 347541

149. Krause, Dr. Peter, o. Professor, Weinbergstr. 12, 5501 Korlingen, (0 6588 ) 7333 (Universität Trier)

150. Krawietz, Dr. Wemer, o. Professor, Nienborgweg 29, 4400 Münster, (02 51) 8614 51; Univ., (02 51) 832591 
151. Krebs, Dr. Walter, Professor,

Lortzingstr. 3, 4800 Bielefeld 1, (05 21) 69739

Universität Bielefeld, Postfach 86 40, 4800 Bielefeld 1, (05 21) 106-43 86

152. Kriele, Dr. Martin, o. Professor,

Richard-Wagner-Str. 10, 5090 Leverkusen 1, (02 14) 5 15 64;

Universität Köln, (02 21) 4702230

153. Kröger, Dr. Klaus, Professor, Hölderlinweg 14, 6300 Gießen-Wieseck, (06 41) 52240

154. Krüger, Dr. Hartmut, Privatdozent,

Freiherr-vom-Stein-Str. 5, 8400 Regensburg, (09 41) 354 06;

Univ., (09 41) 9432659

155. Krüger, Dr. Herbert, o. Professor,

Philosophenweg 14, 2000 Hamburg 50, (0 40) 8807934

156. Küchenhoff, Dr. Erich, Professor,

Dachsleite 65, 4400 Münster, (02 51) 2472 71;

Univ., (02 51) 8327 06/05

157. Kühne, Dr. Jörg-Detlef, Professor,

Eichenweg 5, 5204 Lohmar 1, (0 22 46) 25 50;

Universität Köln, (02 21) 4703834

158. Kunig, Dr. Philip, Privatdozent,

Schlüterstr. 44, 2000 Hamburg 13, (0 40) 4501 21;

Univ. Hamburg, Rothenbaumchaussee 21-23, 2000 Hamburg 13, (0 40) $41234601 / 4604$

159. Lange, Dr. Klaus, Professor,

Lilienweg 22, 6302 Lich, (0 64 04) 56 81;

Universität Gießen, (06 41) 7025019

160. Laubinger, Dr. Hans-Werner, Professor,

Philipp-Wasserburg-Str. 45, 6500 Mainz-Gonsenheim, (0 6131$) 43191$

Universität Mainz, Saarstr. 21,(061 31) 395942

161. Laurer, DDr. Hans René, Universitätsprofessor,

Scheffergasse 27a, A-2340 Mödling, (0 26 36) 204 02;

Universität Wien, (02 22) 3475 44/419

162. Lecheler, Dr. Helmut, o. Professor,

Würzburger Str. 10d, 8600 Bamberg, (09 51) 541 46;

Universität Erlangen, (0 91 31) 854781

163. Leisner, Dr. Walter, o. Professor,

Kochstr. 2, 8520 Erlangen, (0 91 31) 852259 
164. Lerche, Dr. Peter, o. Professor, Junkersstr. 13, 8035 Gauting b. München, (0 89) 85020 88; Universität München, (0 89) 21803335

165. Link, Dr. Heinz-Christoph, Professor, Michaelisweg 4, 3400 Göttingen, (05 51) 486 57; Univ., (05 51) 394693

166. Lipphardt, Dr. Hanns-Rudolf, Professor, Zur Forstquelle 3, 6900 Heidelberg, (0 62 21) 3823 12; Univ., (0 62 21) 547440

167. Listl, Dr. Joseph, o. Professor, Universitätsstr. 10, 8900 Augsburg,(08 21) 598720 od. 598730

168. Löwer, Dr. Wolfgang, Professor, Lotharstr. 3, 5300 Bonn 1, (02 28) 218273

169. Lorenz, Dr. Dieter, o. Professor, Bohlstr. 21, 7750 Konstanz 18, (0 75 33) 68 22; Univ., (0 75 31) 882530

170. Loschelder, Dr. Wolfgang, Professor, Am Ehrenmal 8, 5205 St. Augustin 3, (0 22 41) 3123 16; Univ. Bochum, (02 34) 70052 63/7

171. Magiera, Dr. Siegfried, o. Professor, Feuerbachstr. 1, 6725 Römerberg, (0 62 32) 84454 Hochschule fur Verwaltungswissenschaften Speyer, Freiherr-vom-Stein-Str. 2, 6720 Speyer, (0 62 32) 910348 oder 910331

172. Majer, Dr. Diemut, Professorin, Welfenstr. 30, 7500 Karlsruhe, (07 21) 816650 oder (0 78 41) 41 12;

Fachhochschule des Bundes für öff. Verw. - Fachbereich Bundeswehrverw. - Seckenheimer Landstr. 8-10, 6800 Mannheim 25, (06 21) 408091

173. v. Mangoldt, Dr. Hans, Professor, Goetheweg 1, 7401 Nehren, (0 74 73) 79 08; Universität Tübingen, (0 70 71) 293302

174. Mantl, Dr. Wolfgang, o. Universitätsprofessor, Wiener Str. 256/XI/33, A-8051 Graz, (03 16) 613 06; Univ., (03 16) $31581 /$ Nbst. 479

175 Marti, Dr. Hans, a. o. Professor, Waldriedstr. 29, CH-3074 Bern, (0 31) 5212 66; dienstl., (0 31) 221683 
176. Maunz, Dr. Theodor, o. Professor, Hartnagelstr. 3, 8032 Gräfelfing b. München, (0 89) 8543985 Universität München

177. Maurer, Dr. Hartmut, o. Professor, Säntisblick 10, 7750 Konstanz 19, (0 7533$) 13$ 12;

Univ., (0 75 31) 883657

178. Mayer, Dr. iur. Dr. rer. pol. Heinz, a. o. Universitätsprofessor, An der Goldenen Stiege 10/13, A-2340 Mödling, (0 2236$) 608$ 33;

Universität Wien, (02 22) 430031 22, Schottenbastei 10-16, A-1010 Wien

179. Mayer-Tasch, Dr. Peter Cornelius, Professor, Am Seeberg 11, 8919 Schondorf, (0 81 92) 6 68; Universität München, (0 89) 218030 20/1

180. Meder, Dr. Walter, o. Professor, Buchweiler Str. 20, 1000 Berlin 33, (0 30) 8311289

181. Meessen, Dr. Karl Matthias, Professor, Zobelstr. 18, 8900 Augsburg, (08 21) 5559 89;

Univ., (08 21) 5984 79/2 55

182. Meissner, Dr. Boris, o. Professor, Kleine Budengasse 1, 5000 Köln 1, (02 21) 239754

183. Melichar, Dr. Erwin, o. Universitätsprofessor, Schulerstr. 20, A-1010 Wien;

Univ., (02 22) 43002268

184. Menger, Dr. Christian-Friedrich, o. Professor, Piusweg 108, 4400 Münster, (02 51) 2787 73; Univ., (02 51) 832741

185. Merten, Dr. iur. Dr. rer. pol. Detlef, o. Professor, Von-Dalberg-Str. 8, 6731 St. Martin, (0 63 23) 18 75; Hochschule Speyer, (0 62 32) 910349

186. Meyer, Dr. Hans, Professor, Georg-Speyer-Str. 28, 6000 Frankfurt/M., (0 69) 7757 94;

Univ., (0 69) 7983863

187. Meyn, Dr. Karl-Ulrich, Professor, Schäferweg 2, 4500 Osnabrïck, (05 41) 126482 Universität Osnabrück, (05 41) 6 08-61 36/61 72

188. Mößle, Dr. Dr. Wilhelm, o. Professor, Schwindstr. 19, 8580 Bayreuth, Univ., (09 21) 552866 
189. Morscher, Dr. Siegbert, Universitätsprofessor, Tschiggy freystr. 11a, A-6020 Innsbruck, (0 52 22) 86210

190. Mosler, Dr. Dr. h. c. Hermann, Professor, Mühltalstr. 117a, 6900 Heidelberg, (0 62 21) 480381

191. Müller, Dr. Friedrich, o. Professor, Von der Tann Str. 15, 6900 Heidelberg 1; Univ., (0 62 21) 547481

192. Müller, Dr. Georg, o. Professor, Sugenreben 356, CH-5015 Untererlinsbach, (0 64) 3438 73; Universität Zürich, (01) 25730 03/4

193. Müller, Dr. Jörg Paul, o. Professor, Kappelenring 42a, CH-3032 Hin terkappelen, (0 31) 3605 70; Universität Bern, (0 31) 6588 94/5

194. Müller-Volbehr, Dr. Jörg, Professor, Universitätsstr. 6, 3550 Marburg; Univ., (0 64 21) 283810

195. Münch, Dr. Fritz, apl. Professor, Zur Forstquelle 2, 6900 Heidelberg, (0 62 21) 335 99; Univ., (0 62 21) 42133

196. v. Münch, Dr. Ingo, Professor, Hochrad 9, 2000 Hamburg 52, (0 40) 8296 24; Univ., (0 40) 41234601

197. Murswiek, Dr. Dietrich, Privatdozent, Fasanenweg 6, 6602 Dudweiler, (0 68 97) 7611 51; Univ. des Saarlandes, 6600 Saarbrücken 11, (06 81) 302-2104 u. 3104

198. Mußgnug, Dr. Reinhard, o. Professor, Keplerstr. 40, 6900 Heidelberg, (0 62 21) 462 22; Univ., (0 62 21) 547466

199. v. Mutius, Dr. Albert, o. Professor, Hof Altwasser, 2372 Brekendorf, (0 43 53) 5 15;

Universität Kiel, (04 31) 8803536

200. Nicolaysen, Dr. Gert, Professor, Bockhorst 68a, 2000 Hamburg 55, (0 40) 8701747 ; Univ., (0 40) 41234588

201. Novak, Dr. Richard, o. Universitätsprofessor, Thadd. Stammel-Str. 8, A-8020 Graz, (03 16) 535 16; Univ., (03 16) 3 15 81/4 80 
202. Obermayer, Dr. Klaus, o. Professor, Niendorfstr. 25, 8520 Erlangen, (091 31) 55106

203. Oberndorfer, Dr. Peter, o. Universitätsprofessor, Wolfauerstr. 94, A-4045 Linz, (0 72 22) 349694

204. Öhlinger, Dr. Theo, o. Universitätsprofessor, Tolstojgasse 5/6, A-1130 Wien, (02 22) 821260 ; Univ., (02 22) 430031 44, Schottenbastei 10-16, A-1010 Wien

205. Oldiges, Dr. Martin, Professor, Am Vossberge 6, 4800 Bielefeld, (05 21) 1218 32; Univ., (05 21) 1064399

206. Olshausen, Dr. Henning, o. Professor, Frankenstr. 4, 6710 Frankenthal; Universität Mannheim, (06 21) 2 92-55 97/56 31

207. Oppermann, Dr. Dr. h. c. Thomas, o. Professor, Burgholzweg 122, 7400 Tübingen, (0 70 71) 495 33;

Univ., (0 70 71) 292560

208. Ossenbühl, Dr. Fritz, Professor, Laurentiusstr. 50c, 5330 Königswinter 1, (0 22 23) 41 14;

Universität Bonn, (02 28) 7355 72/3

209. Papier, Dr. Hans-Jürgen, o. Professor, Neusiedler Weg 14, 4904 Enger, (0 52 24) 52 02;

Universität Bielefeld, (05 21) 1064398

210. Partsch, Dr. Karl Josef, o. Professor, Frankenstr. 10, 6507 Ingelheim, (0 61 32) 2264 Universität Bonn

211. Peine, Dr. Franz-Joseph, Professor, Stennerstr. 38, 4800 Bielefeld 1, (05 21) 8868 96; Univ. Hannover, Hanomagstr. 8, 3000 Hannover 91, (05 11) 4738227

212. Pernthaler, Dr. Peter, Universitätsprofessor, Philippine-Welser-Str. 27, A-6020 Innsbruck, (0 52 22) 4182 84; Univ., (0 52 22) 33601731

213. Graf von Pestalozza, Dr. Christian, o. Professor, dienstl.: Thielallee 52, 1000 Berlin 33, (0 30) 8383014

214. Pieroth, Dr. Bodo, Professor, Am Erlenkamp 27, 4630 Bochum, (02 34) 7049 64; Univ., (02 34) 7005262 oder 7005252 
215. Pietzcker, Dr. Jost, Professor, Hausdorffstr. 95, 5300 Bonn, (02 28) 2339 54; Univ., (02 28) 739177

216. Pirson, Dr. Dietrich, o. Professor, Agnesstr. 46, 8000 München 40,(0 89) 27167 77;

Univ., (0 89) 21802715

217. Podlech, Dr. Dr. Adalbert, Professor, Vorm Heiligen Kreuz 2, 6101 Weiterstadt, (0 61 50) 4344 (TH Darmstadt)

218. Püttner, Dr. Günter, o. Professor, Mörikestr. 21, 7400 Tübingen, (0 70 71) 663 94; Univ., (0 70 71) 295262 oder 295263

219. Quaritsch, Dr. Helmut, o. Professor, Otterstadter Weg 139, 6720 Speyer, (0 62 32) 326 37; Hochschule, (0 62 32) 106348

220. Rack, Dr. Reinhard, a. o. Universitätsprofessor, Obere Teichstr. 19, A-8010 Graz, (03 16) 4388 42; Univ., (03 16) 31581 Nbst. 474

221. Randelzhofer, Dr. Albrecht, o. Professor, Van't-Hoff-Str. 8, 1000 Berlin 33, (0 30) 8382288

222. Raschauer, Dr. Bernhard, a. o. Universitätsprofessor, Pfeilgasse 7, A-1080 Wien, (02 22) 43943 02;

Univ., (02 22) 430031 23, Schottenbastei 10-16, A-1010 Wien

223. Rasenack, Dr. Christian A.L., Professor, Dahlemer Weg 63a, 1000 Berlin 37, (0 30) 81737 96;

Techn. Univ., (0 30) 31458 74/75

224. Rauschning, Dr. Dietrich, o. Professor, Rodetal 1, 3406 Bovenden, (0 55 94) 331 Universität Göttingen

225. Rengeling, Dr. Hans-Werner, Professor, Brüningheide 192, 4400 Münster, (02 51) 2120 38; Universität Osnabrück, (05 41) 6086117

226. Ress, Dr. jur. Dr. rer. pol. Georg, o. Professor, Am Botanischen Garten 6, 6600 Saarbrücken, (06 81) 3023055 ;

Univ., (06 81) 3022503

227. Rhinow, Dr. René A., o. Professor, Jurastr. 48, CH-4411 Seltisberg, (0 61) 9123 09; Universität Basel, (0 61) 255277 
228. Riedel, Dr. Eibe H., Professor,

Hinter der Hecke 3, 6501 Nieder-Olm, (0 61 36) 429 91;

Universität Mainz, Saarstr. 21, (061 31) 395759

229. Rill, Dr. Heinz Peter, o. Universitätsprofessor,

Peter-Jordan-Str. 145, A-1190 Wien, (02 22) 47576 15;

Wirtschaftsuniversität, (02 22) 3475 41/2 64

230. Ringhofer, Dr. Kurt, o. Universitätsprofessor, Eduard-Macheiner-Str. 23, A-5020 Salzburg, (06 62) 44767

231. Roellecke, Dr. Gerd, o. Professor, Kreuzackerstr. 8, 7500 Karlsruhe 41, (07 21) 4917 39;

Universität Mannheim, (06 21) 2922851

232. Ronellenfitsch, Dr. Michael, Professor, Richard-Wagner-Str. 22, 6800 Mannheim 1, (06 21) 4035 69;

Universität Bonn, (02 28) 7391 51/91 50

233. Rudolf, Dr. Walter, o. Professor, Rubensallee 55a, 6500 Mainz 31, (0 61 31) 74 21;

Univ., (061 31) 392412

234. Rüfner, Dr. Wolfgang, Professor,

Hagebuttenstr. 26, 5309 Meckenheim, (0 22 25) 71 07;

Univ. Köln, (02 21) 4702679 oder 4703777

235. Rühland, Dr. Curt, o. Professor,

Dürerstr. 26, 3300 Braunschweig, (05 31) 332116

236. Ruland, Dr. Franz, Professor,

Kälberstücksweg 55, 6380 Bad Homburg, (0 61 72) 311 09;

Univ. Frankfurt a.M. 1, (0 69) 1522219

237. Rumpf, Dr. Helmut, Honorarprofessor, Bismarckallee 27, 5300 Bonn 2, (02 28) 353131

238. Rupp, Dr. Hans Heinrich, o. Professor, Am Marienpfad 29, 6500 Mainz, (0 61 31) 34588

239. Sachs, Dr. Michael, Privatdozent, Kleingedankstr. 9, 5000 Köln 1,(02 21) 3186 90; Univ. Köln, Gyrhofstr. 8c, 5000 Köln-Lindenthal, (02 21) 4702289

240. Saladin, Dr. Peter, o. Professor, Forrerstr. 26, CH-3006 Bern; Universität Bern, (0 31) 448006

241. Salzwedel, Dr. Jürgen, o. Professor, Siebengebirgsstr. 86, 5300 Bonn 3, (02 28) 481710 ; Univ., (02 28) 735580 
242. Sattler, Dr. Andreas, Professor, Ludwig-Beck-Str. 17, 3400 Göttingen, (05 51) 223 40; Univ., (05 51) 397377 u. 397393

243. Schäffer, Dr. Heinz, o. Universitätsprofessor, Große Neugasse 6/14, A-1040 Wien, (02 22) 57696 73; Univ. Salzburg, Weiserstr. 22, A-5020 Salzburg, (06 62) 445 11/3 34

244. Schambeck, Dr. Herbert, o. Universitätsprofessor, Hofzeile 21, A-1190 Wien, (02 22) 3634 94; Universität Linz, (07 32) 31310

245. Schenke, Dr. Wolf-Rüdiger, o. Professor, Beim Hochwald 30, 6800 Mannheim, (06 21) 7442 00; Univ., (06 21) 2925214

246. Scherer, Dr. Joachim, LL.M., Privatdozent, Unterlindau 14, 6000 Frankfurt a.M. 1, (0 69) 7257 37; Univ. Frankfurt a.M. 1, (0 69) 7982779

247. Scheuing, Dr. Dieter H., o. Professor, Hans-Sachs-Str. 97, 8706 Höchberg b. Würzburg, (09 31) 483 31; Univ. Würzburg, (09 31) 31324

248. Schick, Dr. Walter, o. Professor, Strindbergstr. 27, 8500 Nürnberg, (09 11) 5014 22; Univ., (09 11) 5302353

249. Schiedermair, Dr. Hartmut, o. Professor, Univ. Köln, Gottfried-Keller-Str. 2, 5000 Köln 41,(02 21) 4702688 oder 4702364

250. Schindler, Dr. Dietrich, Professor, Lenzenwiesstr. 8, CH-8702 Zollikon, (01) 39141 40; Universität Zürich, (01) 3917118

251. Schlaich, Dr. Klaus, o. Professor, Wolkenburgstr. 2, 5202 St. Augustin 2, (0 22 41) 275 09; Universität Bonn, (02 28) 739125

252. Schlink, Dr. Bernhard, Professor, Endenicher Allee 16, 5300 Bonn 1, (02 28) 6523 58;

Universität Bonn, (02 28) 735574

253. Schlochauer, Dr. Hans-Jürgen, Professor, Blauenstr. 18, 6000 Frankfurt, (0 69) 6758 07; Univ., (0 69) 7983193

254. Schmid, Dr. Gerhard, Professor, Hochwaldstr. 24, CH4059 Basel, (0 61) 5084 25;

Sandoz AG, (0 61) 247830 
255. Schmidt, Dr. Reiner, o. Professor, Bachwiesenstr. 4, 8901 Gessertshausen, (0 82 38) 41 11; Universität Augsburg, (08 21) 598443

256. Schmidt, Dr. Walter, Professor, Brüder-Knauß-Str. 86, 6100 Darmstadt, (0 61 51) 647 10; Universität Frankfurt, (0 69) 7982189

257. Schmidt-Aßmann, Dr. Eberhard, o. Professor, Höhenstr. 30, 6900 Heidelberg, (0 62 21) 8008 03; Univ., (0 62 21) 547428

258. Schmidt-Jortzig, Dr. Edzard, Professor, Graf-Spee-Str. 18a, 2300 Kiel 1; Univ., (04 31) 8 80-35 45

259. Schmitt Glaeser, Dr. Walter, o. Professor, Rübezahlweg 9A, 8580 Bayreuth, (09 21) 32070 ; Univ., (09 21) 552942

260. Schmitt-Kammler, Dr. Arnulf, Professor, Renthof 33, 3550 Marburg/Lahn, (0 64 21) 649 02; Univ. Köln, (02 21) 4703544 oder 4703500

261. Schnapp, Dr. Friedrich E., o. Professor, Efeuweg 22, 4630 Bochum 6, (0 23 27) 742 13; Univ. Bochum, (02 34) 7002239

262. Schneider, Dr. Hans, o. Professor, Ludolf-Krehl-Str. 44, 6900 Heidelberg, (0 62 21) 480381

263. Schneider, Dr. Hans-Peter, Professor, Delpweg 16, 3000 Hannover 91, (05 11) 4671 66;

Univ., (05 11) $7628185 / 6$

264. Schneider, Dr. Litt. D. h. c. Peter, o. Professor, Goldenluftgasse 4, 6500 Mainz, (0 61 31) 223273

265. Schnur, Dr. Roman, o. Professor, Lindenstr. 49, 7407 Rottenburg 5, (0 74 72) 22224 Universität Tübingen

266. Scholler, Dr. Heinrich, Professor, Zwengauerweg 5, 8000 München 71, (0 89) 7964 24; Univ., (0 89) 21802724

267. Scholz, Dr. Rupert, o. Professor, Erbacher Str. 1, 1000 Berlin 33, (0 30) 8911700 Universität München, (0 89) 21802113 
268. Schröder, Dr. Meinhard, o. Professor, Zum Wingert 2, 5501 Mertesdorf, (06 51) 578 87; Universität Trier, (06 51) 2012586

269. Schuppert, Dr. Gunnar Folke, Professor, Beethovenstr. 1, 8900 Augsburg, (08 21) 1512 71; Univ. Augsburg, Eichleitner Str. 30, 8900 Augsburg, (08 21) 5981

270. Schwabe, Dr. Jürgen, Professor, Erlenweg 1, 2150 Buxtehude, (0 41 61) 871 41; Universität Hamburg, (0 40) 41233021

271. Schwarze, Dr. Jürgen, o. Professor, Universität Hamburg, Schlüterstr. 28, 2000 Hamburg 13, (0 40) $41234564 / 4571$

272. Schweitzer, Dr. Michael, Professor, Göttweiger Str. 135, 8390 Passau; Univ., (08 51) 5505547

273. Schwerdtfeger, Dr. Gunther, o. Professor, Thielallee 52, 1000 Berlin 33, (0 30) 8383010

274. Scupin, Dr. Hans Ulrich, o. Professor, Robert-Koch-Str. 46, 4400 Münster, (02 51) 823 41; Univ., (02 51) 832764

275. Seewald, Dr. Otfried, o. Professor, Göttweiger Str. 41, 8390 Passau, Univ., (08 51) 509158 und 509159

276. Seidl-Hohenveldern, Dr. Dr. h. c. Ignaz, o. Professor, A-1010 Wien I, Schwertgasse 4, (02 22) 631560

277. Selmer, Dr. Peter, Professor, Akazienweg 9, 2000 Hamburg 55, (0 40) 8647 43; Univ., (0 40) 41234576

278. Siedentopf, Dr. Dr. h. c. Heinrich, o. Professor, Hauptstr. 170, 6740 Landau-Godramstein, (0 63 41) 607 57; Hochschule Speyer, (0 62 32) 9 10-2 12

279. v. Simson, Dr. Werner, o. Professor, Luisenstr. 3, 7800 Freiburg, (07 61) 35863

280. Skouris, Dr. Wassilios, Professor, Nikolaou Manou 18, 54643 Thessaloniki, Griechenland, (0030 31) 831444 Fachber. Rechtswissensch., (00 3031$) 991389$ 
281. Söhn, Dr. Hartmut, o. Professor, Eppanerstr. 9, 8390 Passau, (08 51) 585 20;

Universität Passau, (08 51) 509192

282. Soell, Dr. Hermann, o. Professor, Domspatzenstr. 34, 8411 Etterzhausen, (0 94 04) 21 25;

Universität Regensburg, (09 41) 9432657

283. Spanner, Dr. Hans, o. Professor, Candidstr. 24, 8000 München 90, (0 89) 652141

284. Staff, Dr. Ilse, Professorin, Am Forum 4, 6233 Kelkheim, (061 95) 3308

285. Starck, Dr. Christian, Professor, Unter den Linden 20, 3400 Göttingen, (05 51) 7926 44;

Univ., (05 51) 397412

286. Steiger, Dr. Heinhard, Professor,

Oberhof 16, 6307 Linden, (06 41) 232 52;

Univ., (06 41) 7025030

287. Stein, Dr. Ekkehart, Professor, Jakob-Burckhardt-Str. 49, 7750 Konstanz, (0 75 31) 632 57;

Univ., (0 75 31) 882329

288. Stein, Dr. Torsten, Privatdozent,

Ludolf-Krehl-Str. 1b, 6900 Heidelberg 1, (0 62 21) 4804 38;

dienstl., (062 21) 482230

289. Steinberg, Dr. Rudolf, Professor,

Senckenberganlage 31, 6000 Frankfurt/M. 1;

Univ., (0 69) 7982438

290. Steinberger, Dr. Helmut, o. Professor,

Schloß West 140, 6800 Mannheim, (0 62 21) 369 54;

dienstl., (06 21) 2923368

291. Steiner, Dr. Udo, 0. Professor,

Am Katzenbühl 5, 8400 Regensburg-Harting, (0 94 01) 43 13;

Univ., (09 41) $9432666 / 7$

292. Stern, Dr. Klaus, o. Professor, Universität Köln, Gyrhofstr. 8c, 5000 Köln 41, (02 21) 4702289

293. Stettner, Dr. Rupert, Professor, Jahnstr. 6, 8060 Dachau, (0 81 31) 132 44; Universität Bamberg, Postfach 15 49, 8600 Bamberg, (09 51) 793321 oder 793318 
294. Stober, Dr. Rolf, Professor,

Hohe Geist 32, 4400 Münster, (0 25 36) 17 34;

Univ., (02 51) 832704

295. Stock, Dr. Martin, Professor,

Am Knick 22, 4800 Bielefeld 1, (05 21) 8895 33;

Univ., (05 21) 1064382

296. Stödter, Dr. Rolf, Professor,

Golfstr. 7, 2057 Wentorf b. Hamburg, (0 40) 7202646

297. Stolleis, Dr. Michael, o. Professor,

Waldstr. 15, 6242 Kronberg 2,

Universität Frankfurt a.M., (0 69) 7983192

298. Stolzlechner, Dr. Harald, Universitätsdozent,

Sackengutstr. 5b, A-5020 Salzburg, (06 62) 4202 52;

Univ., (06 62) 44511

299. Suhr, Dr. Dieter, Professor,

Birkenstr. 37, 8900 Augsburg 22, (08 21) 976 46;

Univ., (08 21) 598355

300. Tettinger, Dr. Peter J., o. Professor,

Bergstr. 30, 5000 Köln 50, (0 22 36) 668 56;

Universität Bochum, (02 34) 7005275

301. Thieme, Dr. Werner, Professor,

Am Karpfenteich 58, 2000 Hamburg 63, (0 40) 53849 92;

Univ., (0 40) 41232627

302. Thürer, Dr. Daniel, Professor,

Abeggweg 20, CH-8057 Zürich, (01) 3626547

Univ., (01) 2573118

303. Tomuschat, Dr. Christian, Professor,

Kautexstr. 43, 5300 Bonn 3,(02 28) 4300 67;

Universität Bonn, (02 28) 739172

304. Trzaskalik, Dr. Christoph, Professor,

Im Damm 1, 6531 Ockenheim, (0 67 25) 45 21;

Universität Mainz, (0 61 31) 392138

305. Tsatsos, Dr. Dimitris Th., o. Professor, Am Waldesrand 10e, 5800 Hagen, (0 23 31) 5866 68;

Fernuniv., (0 23 31) 8042876

306. Uber, Dr. Giesbert, o. Professor,

Roseneck 5, 4400 Münster-Hiltrup, (0 25 01) 31 59;

Univ., (02 51) 832701 
307. Ule, Dr. Carl Hermann, o. Professor, Oberer Gaisbergweg 9, 6900 Heidelberg, (0 62 21) 278 32; Hochschule Speyer

308. v. Unruh, Dr. Georg-Christoph, o. Professor, Steenkamp 2, 2305 Heikendorf, (04 31) 2314 59; Universität Kiel, (04 31) 88035 22/69

309. Graf Vitzthum, Dr. Wolfgang, o. Professor, Herderstr. 12, 7410 Reutlingen 1, (0 71 21) 2402 05; Univ. Tübingen, (0 70 71) 295266

310. Vogel, Dr. Klaus, o. Professor, Ottostr. 12, 8130 Stamberg, (0 81 51) 132 21; Universität München, (0 89) 21802718

311. Voigt, Dr. Alfred, o. Professor, Schwedenstr. 26, 8521 Spardorf, (0 91 31) 56043

312. Wagner, Dr. Heinz, o. Professor, Tietzenweg 54, 1000 Berlin 45, (0 30) 83321 67; Univ., (0 30) 8383639

313. Wahl, Dr. Rainer, o. Professor, Sundgauallee 68, 7800 Freiburg, (07 61) 858 71; Univ., (07 61) 20344 65/6

314. Weber, Dr. Albrecht, Professor, Weidenweg 20, 4516 Bissendorf, (0 54 02) 39 07; Universität Osnabrück, (05 41) 6 08-61 88

315. Weber-Dürler, Dr. Beatrice, Privatdozentin, Susenbergstr. 5, CH-8044 Zürich, (01) 690420

316. Wehrhahn, Dr. Herbert, o. Professor, Lyckallee 42, 1000 Berlin 19

317. Weides, Dr. Peter, o. Professor, Franz-Marc-Str. 22, 5000 Köln 50, (02 21) 3911 92; Univ., (02 21) 4704454

318. Wendt, Dr. Rudolf, Professor, Caspar-Olevian-Str. 57, 5500 Trier, (06 51) 380 47; Universität Trier, Postfach 38 25, (06 51) 20125 76/77

319. Wenger, DDr. Karl, Universitätsprofessor, Meytensgasse 18, A-1130 Wien, (02 22) 82272 44; Univ., (02 22) 430031 36, Schottenbastei 10-16, A-1010 Wien

320. Wengler, Dres. Dres. h. c. Wilhelm, Professor, Werderstr. 15, 1000 Berlin 37, (0 30) 8016535 
321. Wertenbruch, Dr. Wilhelm, Professor, An der Rodung 6, 5353 Mechernich-Katzvey, (0 22 56) 78 18; Universität Bochum, (02 34) 7002239

322. Wielinger, Dr. Gerhart, Universitätsdozent, Bergmanngasse 22, A-8010 Graz, (03 16) 318714 dienstl., (03 16) 70312428

323. Wildhaber, Dr. Luzius, o. Professor, Auf der Wacht 21, CH-4104 Oberwil, (0 61) 302521

324. Wilke, Dr. Dieter, o. Professor, Universität Berlin, Thielallee 52, 1000 Berlin 33, (0 30) 8383011

325. Wimmer, Dr. Norbert, o. Universitätsprofessor, Claudiastr. 7, A-6020 Innsbruck, (0 52 22) 204 27; Univ., (0 52 22) $33601 / 731$

326. Winkler, Dr. Günther, Universitätsprofessor, Reisnerstr. 22/5/11, A-1030 Wien, (02 22) 7344 15; Univ., (02 22) 430031 31, Schottenbastei 10-16, A-1010 Wien

327. Wolfrum, Dr. Rüdiger, o. Professor, Lindenallee 13, 2300 Kiel 1 (Altenholz), (04 31) 32 18 44; Univ., (04 31) 8 80.21 89

328. Wollenschläger, Dr. Michael, Privatdozent, An den Forstäckern 15, 8706 Höchberg, (09 31) 49196 Univ., (09 31) 31305

329. Würtenberger, Dr. Thomas, o. Professor, Im Brühl 9, 5501 Gutweiler, (0 65 88) 71 79;

Universität Trier, (06 51) 716451

330. Zacher, Dr. Hans F., o. Professor, Stamberger Weg 7, 8134 Pöcking, (0 81 57) 13 84; Universität München, (0 89) 21802725

331. Zeh, Dr. Wolfgang, Ministerialrat, Sibyllenstr. 40, 5300 Bonn 2, (02 28) 3756 52; dienstl., (02 28) 162649 oder 163044

332. v. Zezschwitz, Dr. Friedrich, Professor, Petersweiher 47, 6300 Gießen, (06 41) 451 52; Univ., (06 41) 7025020

333. Zieger, Dr. Gottfried, Professor, Leuschnerweg 10, 3400 Göttingen, (05 51) 22255

334. Zimmer, Dr. Gerhard, Privatdozent, Bamberger Str. 22, 1000 Berlin 30, (0 30) 8544656 
335. Zippelius, Dr. Reinhold, o. Professor,

Niendorfstr. 5, 8520 Erlangen, (0 91 31) 557 26;

Univ., (091 31) 852820

336. Zuleeg, Dr. Manfred, Professor,

Kaiser-Sigmund-Str. 32, 6000 Frankfurt/M. 1, (0 69) 5643 93;

Univ., (0 69) 7982382 